\title{
Computer Simulation of Heart-Pacemaker Interaction
}

\author{
Saul E. Greenhut, MS, Robert S. MacDonald, MSE, and \\ Janice M. Jenkins, PhD
}

The electrical interaction between the heart and an artificial pacemaker is often complex. Because of the sophistication and variability of dual-chamber device algorithms, even experienced cardiologists have difficulty interpreting paced electrocardiograms (ECGs). To study heart-pacemaker interaction (HPI), a computer model of the cardiac conduction system has been developed which includes the effects of artificial pacemaker function and failure.

The cardiac conduction model, based upon automata theory, consists of nine vertices each representing a functional electrophysiological element. Connections between vertices include normal and accessory, anterograde and retrograde pathways. Electrophysiological multidimensional conditional probability functions determine the depolarization status of each vertex. A depolarization event occurs at a vertex if its probability of occurrence at time $t$ is greater than a pseudorandom number. The atrioventricular (AV) node is emulated using a mathematical model that includes the influence of past cycle lengths on AV nodal conduction time. Twentyfive classes of arrhythmias may be simulated and any one of 12 antibradycardia pacing modes and 6 electrophysiology study protocols may be chosen.

From the Department of Electrical Engineering and Computer Science and the Bioengineering Program. The University of Michigan, Ann Arbor, Michigan.

Supported in part by NSF Grant BCS-8909042 and a gift from Telectronics, Inc.

Reprint requests: Saul E. Greenhut, MS, Medical Computing Laboratory, Department of Electrical Engineering and Computer Science, University of Michigan, Ann Arbor, MI 48109-2122.
Random effects of pacemaker malfunction due to oversensing, undersensing, or failure to capture may be simulated. Amplitudes of spontaneous atrial and ventricular depolarizations are Gaussian distributed, with the mean and standard deviation being selected for individual hearts. Gaussian noise, such as from a myopotential source, is also specified to occur at random intervals. Once these distributions are set, pacemaker atrial and ventricular sensitivites are selected to discriminate cardiac depolarization from noise. Oversensing or undersensing may be simulated by varying the depolarization distribution, noise distribution, or sensitivity values. Failure to capture is simulated by specifying a Gaussian-distributed myocardial stimulation threshold level, as well as pacemaker pulse amplitude and width. Pacing energy is compared with a random Gaussian number representing the stimulation threshold. If output energy exceeds threshold, capture of the appropriate chamber occurs; otherwise there is failure. Computer output consists of continuously generated ladder diagrams in which idealized normal, ectopic, and paced depolarization morphologies are differentiated. The graphical output also includes a pacemaker marker channel that indicates pacemaker response and noise.

This model should prove useful in development of pacemaker algorithms, determining patient-specific pacemaker therapy, and predicting causes for apparent pacemaker malfunction. Currently, the model is being used in the development of an expert system to analyze paced ECGs for pacemaker function/malfunction and arrhythmia. 